Psicologia \& Sociedade; 20 (2): 277-286, 2008

\title{
EL PREJUICIO RACIAL EN BRASIL: NUEVAS MEDIDAS Y PERSPECTIVAS
}

\author{
Ana Maria Lacerda Teixeira Pires \\ Fernando Molero Alonso \\ Universidad Nacional de Educación a Distancia, Madrid, España
}

\begin{abstract}
RESUMEN: Se realizaron dos estudios. El primer estudio trataba de comprobar si la escala de racismo moderno de McConahay, Hardee y Batts (1981), resultaba un instrumento válido en la medición de las actitudes prejuiciosas en una muestra de la población brasileña. Se aplicó los cuestionarios a un total de 206 estudiantes de dos colegios. Los datos obtenidos muestran una fiabilidad aceptable de la escala. Los resultados muestran que el prejuicio declarado hacia los afrobrasileños es relativamente bajo. El segundo estudio analizó la percepción general de prejuicio y discriminación en una muestra de la población negra/mestiza. Los resultados muestran una moderada percepción general de prejuicio hacia su grupo y la frecuencia en la que han sido discriminados personalmente ha sido baja. Encontramos diferencias entre la variable etnia y discriminación personal percibida.
\end{abstract}

PALABRAS CLAVE: Racismo moderno; prejuicio; discriminación.

\section{RACIAL PREJUDICE IN BRAZIL: NEW MEASURES AND PERSPECTIVES}

ABSTRACT: Two studies were performed. The first study was done in order to verify the validity of the scale of modern racism proposed by McCohahay, Hardee and Batts (1981) to measure the prejudiced attitudes in a sample of the Brazilian population. Questionnaires were applied in 206 students from two schools. The obtained data showed an acceptable reliability of the scale. Results show that the expression of prejudice declared by the African-Brazilians is relatively low. The second study examined the general perception of prejudice and discrimination on a sample of a black/mestizo population. The results show a moderate general perception of prejudice with this group but the frequency which they had been personally discriminated was low. We found differences between different ethnic groups and perceived personal discrimination.

KEYWORDS: Modern racism; prejudice; discrimination.

\section{Nuevas Formas del Prejuicio Racial}

Una cuestión planteada en la investigación reciente sobre el tema del racismo es la de que existen nuevas formas de expresar éste, de manera indirecta y sutil, en contraste con las formas abiertas. Estas parecen estar siendo sustituidas por actitudes menos evidentes, que reproducen actitudes discriminatorias, sin entretanto, confrontar las normas sociales de la indeseabilidad del racismo.

Las nuevas teorías acerca del prejuicio a partir de la década de los setenta, como la del racismo simbólico (Kinder \& Sears, 1981), del racismo moderno (McConahay, 1986) del racismo ambivalente (Katz \& Hass, 1988) y la del prejuicio sutil (Pettigrew \& Meertens, 1995) describen procesos discriminatorios menos flagrantes y más encubiertos.

En los Estados Unidos, el racismo simbólico representa una forma de resistencia a cambios en el status quo de las relaciones raciales. Se basa en sentimientos y creencias de que los negros violan los valores tradicionales del individualismo y de la ética protestante (Kinder \& Sears, 1981). Según estos autores se trata de la percepción por parte del grupo dominante de que los negros representan más una amenaza simbólica para los valores de la cultura de dicho grupo que propiamente una amenaza económica concreta. El concepto de racismo moderno de la misma manera que el concepto del racismo simbólico (la expresión de esta idea está basada en símbolos ideológicos abstractos), refleja la percepción de que los negros están consiguiendo más de lo que merecen y violando valores importantes para los blancos. De la necesidad empírica de medir las actitudes raciales de los individuos, surgió la teoría del racismo moderno. Esta teoría ha sido desarrollada juntamente con la Escala de Racismo Moderno (McConahay \& Hough Jr., 1976).

El racismo moderno también ha sido investigado en otros contextos además de los Estados Unidos, utilizando otras clases de muestras. Pedersen y Walker (1997) han estudiado el prejuicio hacia los aborígenes en Australia, 
con el objetivo de analizar la relación entre el racismo moderno y el racismo flagrante. En estos estudios han utilizado una adaptación de la escala de racismo moderno propuesta por Augoustinos, Ahrens y Innes (1994) y una escala de racismo flagrante propuesta por Walker (1994). En un otro estudio, Navas (1998), en el que tuvo como objetivo adaptar a la población española la escala de racismo moderno de McConahay et al. (1981), ha utilizado una muestra de inmigrantes.

Estas líneas de investigación han generado un enorme cuerpo de datos, pero la utilización de estos conceptos no está exenta de críticas (Rueda \& Navas, 1996). Aunque existan algunas diferencias conceptuales y metodológicas entre los distintos autores, los resultados de estos trabajos demuestran que existe una nueva expresión del prejuicio y que se caracteriza por sentimientos y creencias discriminatorias que adquieren matices distintos del "viejo racismo". No obstante las diferencias que existan entre las nuevas teorías del racismo, el aspecto común entre ellas, es el hecho de que no se trata de una reducción cuantitativa en las expresiones del prejuicio, sino de un cambio cualitativo de dichas expresiones. Estos abordajes sugieren que la disminución de las expresiones del racismo sería más aparente que real.

De acuerdo con Wetherell y Potter (cit. en Echebarría \& Villareal, 1995, p. 210) aunque el discurso racista va evolucionando y puede adoptar diversas formas, lo importante es que cumple una función social clara: establecer, sostener y reforzar relaciones de poder opresivas. Es decir, es un discurso que justifica, sostiene y legitima prácticas orientadas a mantener el poder y la dominación de unos grupos sobre otros. Por esta razón las definiciones anteriores (racismo simbólico, sutil, moderno o aversivo) que caracterizan el racismo como un conjunto de pensamientos y sentimientos negativos sobre grupos, no pueden explicar el caráscter cambiante del discurso racista o el recurso a principios progresistas para justificar la exclusión de ciertos grupos. Desde esta perspectiva, el racismo es la expresión de ciertas relaciones de poder cuyas causas se sitúan en una estructura social de dominación, por parte de ciertos grupos y de opresión de otros grupos.

La contextualización histórica del racismo propuesta por Duckitt (1992) nos da una idea deque las definiciones del prejuicio y del racismo reflejan el entorno social y histórico de dichas relaciones raciales. Así la naturaleza y las formas de manifestación del prejuicio se encuentran bajo la influencia y normas sociales del un contexto definido.

\section{Contextualización del}

\section{Prejuicio Racial en Brasil}

Como hemos señalado anteriormente, aunque se estudie el racismo como una actitud, se torna evidente que las condiciones históricas, culturales y económicas estén ligadas a comportamientos específicos. Los estudios citados anteriormente, los cuales han sido realizados en los Estados Unidos y en países europeos ponen de relieve el carácter distinto de las relaciones interétnicas entre ellos, incluso por las relaciones históricas entre los diversos grupos. En dichos contextos la definición se hace por la percepción de los exogrupos como en Europa y en la diferenciación con base en la ascendencia racial como es el caso en los Estados Unidos. ¿Pero como se expresa el racismo en un entorno social con características multirraciales y multiétnicas como es el caso de la sociedad brasileña?

Freyre (1933), ha considerado que el proceso histórico de mezcla racial en Brasil, fue un legado positivo que ha contribuido a la armonía de las relaciones raciales existentes. No obstante, la ideología de la "democracia racial", defendida por el autor, ha sido criticada y definida como "mito" al ser contrastada más tarde con las desigualdades sociales existentes en la sociedad brasileña (Bastide \& Fernandes, 1959).

Estudios realizados sobre el prejuicio racial, en los años 50, destacaron la distancia social entre blancos y negros, medidos por la "escala de distancia social de Bogardus" (Guimarães, 1999, p. 90). Estas investigaciones han demostrado los problemas de interacción entre estos grupos además de las dificultades de ascenso social experimentadas por los negros y mestizos en distintas regiones del país. Los resultados muestran que los entrevistados, no dudaban en afirmar abiertamente sus prejuicios y evidenciaban el rechazo a una mayor posibilidad de proximidad social (Bastide, 1956; Bastide \& van den Berghe, 1957). Para varios autores, los resultados de estas investigaciones mostraron la discrepancia entre la adhesión a los valores de igualdad, a las normas democráticas, la aceptación de la igualdad de oportunidades para blancos y negros por un lado, y por otro, el alto grado de estereotipos negativos y la segregación en las relaciones personales por parte de los blancos con relación a los negros y mestizos. En un otro importante estudio realizado por Fernandes y Bastide (1959), estos autores concluyeron que los brasileños no evitaban el prejuicio, pero se avergonzaban de ser prejuiciosos, porque consideraban "feo"(malo) admitir la discriminación, lo que Fernandes ha denominado de "prejuicio retroactivo". Para este autor, "no se hablaba, no se demostraba pero se reconocía la existencia". De esta manera, "las jerarquías permanecían intocables y lo que uno exteriorizaba era la amabilidad". A su vez, Bastide (1956) ha destacado la influencia del mito de la democracia racial en las "no manifestaciones abiertas del prejuicio racial" y en el "problema racial estar disimulado entre las clases sociales". Debido a esto, las barreras encontradas por los negros se disimulaban en un ritual de amabilidad que aumentaba la distancia social en lugar de disminuirla. 
Autores más recientes como Maggie y Gonçalves (1995, p. 8) reconocen que en el presente tenemos un sistema de relaciones raciales que colaboran con la división y clasificación de los grupos de acuerdo con sus diferencias y cuestionan el mito de la democracia racial como responsable por la negación del racismo.

Peter Fry (2005), por otro lado, critica aquellos que quieren enterrar la idea de la "democracia racial", argumentando que esta ya es parte de la sociedad brasileña, igual que las prácticas discriminatorias y lleva consigo valores e ideales positivos los cuales se debería cultivar.

Estos cuestionamientos nos dejan percibir las peculiaridades de la cuestión racial y el modo complejo de interacción que se ha creado.

La creciente preocupación de los investigadores con respecto a las desigualdades raciales y el mayor número de estudios mejor documentados que se desarrollaron en las últimas décadas no sólo no corroboran las ideas optimistas acerca de las relaciones raciales en Brasil (Fernandes, 1972; Nascimento, 1968) sino que han demostrado que el mito de la democracia racial parece ser una forma de racionalización de las prácticas discriminatorias (Azevedo, 1975). Por su parte, los movimientos negros en los años setenta, también han criticado la falsa realidad que presupone la "democracia racial", denunciando el racismo y reivindicando una cultura y identidad negra (Hasenbalg \& Silva, 1992).

Turra e Venturi (1995), en sus estudios describen un tipo de racismo típicamente brasileño, el "racismo cordial". El racismo cordial lo definen como una forma de discriminación en contra los ciudadanos no blancos (negros y mestizos), que se caracteriza por una cortesía superficial que encubre actitudes y comportamientos discriminatorios y que se expresa en las relaciones interpersonales a través de chistes, dichos populares y bromas de carácter racial. El presupuesto empírico de esta teoría resultó de un estudio que fue llevado a cabo en todo el territorio nacional y arrojó que el $89 \%$ de los brasileños reconocía la existencia del prejuicio racial. Aunque la mayoría de los sujetos admita que la sociedad brasileña es prejuiciosa, solo el $10 \%$ se ha considerado personalmente prejuicioso.

Estos autores han desarrollado una escala menos directa para medir el "racismo cordial" y han verificado que más de $50 \%$ de la muestra de los entrevistados estaban de acuerdo con las afirmaciones de dicha escala y uno $83 \%$ de los sujetos declararon estar de acuerdo con algún aspecto de los ítems de la escala de racismo cordial (Turra \& Venturi, 1995).

En un otro estudio, Martinez e Camino (2000), encontraron en esta misma línea que los estudiantes universitarios cuando preguntados por su grado de prejuicio en una escala de 1 a 10 (mayores puntuaciones indicaban mayor atribución de prejuicio) atribuyeron a "si mismos" en una media de 3,3 puntos, mientras que atribuyeron 7,8 puntos a "los brasileños en general".

Así, los resultados de las investigaciones, los estudios realizados por instituciones antiracistas y los organismos oficiales, la implementación de las políticas de acción afirmativa desde mediados de los años noventa, comprueban las desigualdades raciales y asocian la idea del racismo a las oportunidades desiguales entre los grupos.

Los datos de da Síntese de Indicadores Sociais 2005, elaborada por el IBGE (Instituto Brasileiro de Geografia e Estatística) a partir del a PNAD 2004 (Encuesta Nacional por Amostra de Domicílios), han demostrado las diferencias entre la población blanca y la población afrodescendiente en lo que se refiere al mercado de trabajo y la formación escolar. Según estos resultados el salario de los trabajadores negros en Brasil es en promedio 50\% menor que el de los blancos. La desigualdad racial se manifiesta también con relación a la asistencia a la escuela. Los blancos poseen una media de 9,8 años de estudio, lo que representa 2,1 años de estudio más que a población afrodecendiente. La cantidad de investigaciones realizadas no ofrecen dudas, acerca de la percepción del prejuicio racial por parte de la mayoría de los entrevistados y, por otro lado, los indicadores sociales de desigualdades entre los ciudadanos blancos, negros y mestizos ponen de manifiesto dichas diferencias.

Debido a esto, no deja de ser importante que el abordaje de la investigación incluya el estudio de las perspectivas de los colectivos afectados. Así, conocer la opinión del grupo desfavorecido es fundamental para entender el prejuicio así como sus consecuencias.

\section{Definición de Afrodescendiente}

La definición operativa de afrodescendiente varía en los países de América Latina. En cada país, los distintos censos y encuestas han hecho operativa la identificación de los grupos étnicos a través de los criterios de la lengua materna y de la autopertenencia o autoidentificación. En Brasil los afrodescendientes corresponden a la suma de "pretos" (negros) y "pardos" (mulatos y mestizos).

Debe resaltarse que la definición de lo que es afrobrasileño, en su aspecto conceptual es algo sobre lo que no existe consenso y parece llevar tanto a sobreestimaciones como a subestimaciones de la cantidad de población de estos pueblos, dependiendo de los criterios empleados (autodefiniciones o censos yencuestas oficiales). La necesidad de evitar falsas identificaciones y de adaptarse a diferentes clasificaciones, ha hecho con que en este estudio se utilice el criterio básico de oponer los afrodescendientes al resto de la población considerada blanca y utilizando como sinónimo los términos afrobrasileños - negros y mestizos. 
Tabla 1

Distribución de la Población: Cor o Raza en Percentuales

\begin{tabular}{lcccc} 
Regiones & Blanca & Negra & Parda & $\begin{array}{c}\text { Amarilla/ } \\
\text { Indigena** }\end{array}$ \\
\hline Brasil & 54,0 & 5,4 & 39,9 & 0,6 \\
Región Norte* & 28,4 & 2,3 & 68,63 & 1,0 \\
Región Noreste & 29,7 & 5,6 & 64,5 & 0,2 \\
Región Sureste & 64,0 & 6,7 & 28,4 & 0,8 \\
Región Sur & 83,6 & 3,0 & 12,6 & 0,7 \\
Región Centro-Oeste & 46,2 & 3,5 & 49,4 & 0,8 \\
\hline
\end{tabular}

Nota. *Solo la población urbana; **Las dos categorias van juntas (IBGE, 2000).

\section{Objetivos de la Presente Investigación}

Son pocos los estudios empíricos dirigidos a evaluar los distintos modos de percepción del racismo y las nuevas formas que están adquiriendo la manifiestación del prejuicio racial y los estereotipos sobre los negros y mestizos en Brasil. Así, nuestro estudio es un esfuerzo por contribuir al estudio del prejuicio en este entorno social, basándonos teóricamente y metodológicamente en las nuevas teorías sobre el prejuicio racial.

De acuerdo con lo expuesto más arriba, hoy en día se han elaborado nuevas fórmulas sutiles para investigar el prejuicio de las mayorías. Con este propósito McConahay et al. (1981) han desarrollado la escala de racismo moderno, un instrumento menos reactivo que las escalas convencionales para medir el racismo, sostienen los autores.

La escala ha sido aplicada además de los Estados Unidos, en Gran Bretaña (Lepore \& Brow, 1994, cit. en Brown, 1995, cap. 7) y en Australia (Augoustinos, Ahrens \& Innes, 1994) y España (Navas, 1998), como un instrumento fiable para medir el prejuicio.

Por tanto, considerando el contexto que acabamos de describir, el presente estudio tiene como objetivos principales: (a) adaptar la escala de racismo moderno de McConahay et al. (1981) a la población brasileña, a fin de comprobar si dicha escala puede ser considerada como un instrumento válido para medir el prejuicio en este entorno social; (b) analizar las manifestaciones de prejuicio en sujetos blancos, pero no menos importante, conocer la percepción de prejuicio y discriminación en sujetos negros y mestizos forma:

Diseñamos la presente investigación de la siguiente

1. Estudio 1 - Analizar las manifestaciones de prejuicio en sujetos pertenecientes a la raza blanca (autoclasificación).
2. Estudio 2 - Medir el grado de prejuicio general y discriminación personal en los sujetos que se autoidentificaron pertenecientes a los grupos negro y mestizo.

\section{Método}

\section{Estudio 1}

Estudio del Prejuicio en los Sujetos de la Raza Blanca

Muestra

En el primer estudio participaron 105 sujetos de la raza blanca (60 mujeres y 45 hombres). Sus edades oscilan entre 16 y 48 años, con una media de 20,6 ( $S D=5.56)$. Con referencia a la ocupación, el 44,8\% de los sujetos solo estudia y el $55,2 \%$ trabaja y estudia.

\section{Procedimiento y Instrumentos}

Los estudiantes que participaron en este estudio contestaron un cuestionario, en el que, además de indicar la raza de pertenencia, el sexo, edad y ocupación, se incluían las siguientes secciones:

1- Una versión de la escala de racismo moderno de McConahay et al. (1981) para medir el prejuicio hacia los negros y mestizos. Esta escala constaba de 10 ítems, con un formato de respuesta tipo Likert de 7 puntos (1-totalmente en desacuerdo hasta 7-totalmente de acuerdo).

2- $\quad$ La escala de emociones (Rueda \& Navas, 1996), compuesta de 14 sentimientos o emociones entre ellos, seis emociones positivas y ocho negativas en la que los sujetos debían responder utilizando una escala de 5 puntos (1=Nada; 5= Mucho). Se han sustituido algunos ítems de esta escala de acuerdo con los objetivos de la investigación. 
Las personas participantes en la investigación se contactaron en dos colegios de la ciudad de Porto Alegre. El estudio se presentó a los sujetos como una investigación relacionada a la apreciación de diferencias sociales económicas y culturales entre los diversos grupos.

Se les pedía que eligieran el cuestionario correspondiente a la categoría étnica la cual más se autoidentificaban. Este ha sido el procedimiento de autoclasificación en relación a las razas: blanca, negra/ mestiza. Todos los sujetos participaran voluntariamente de la investigación y respondieron los cuestionarios de forma individual y anónima.

\section{Resultados}

\section{Fiabilidad y Estadísticos Descriptivos}

En primer lugar, con el fin de analizar la fiabilidad de la escala de racismo moderno, realizamos análisis de fiabilidad Alpha de Cronbach, obteniéndose un coeficiente de .63. Es algo bajo pero que podemos considerar aceptable.

La media del total de esta escala fue de 3.49. Esta diferencia es significativa $t(104)=-5.25 ; p<0001$ con respecto al punto medio de la escala (4). Por lo que podemos concluir que el prejuicio declarado hacia los afrobrasileños es relativamente bajo. Si analizamos las características de la muestra podemos verificar que ella esta formada por jóvenes estudiantes y de acuerdo con la literatura, estas variables están relacionadas con niveles más bajos de prejuicio. Existen diferencias significativas en función del género de los sujetos. Así, las mujeres declararon sentir menos prejuicio hacia los afrodescendientes que los hombres (3,27 vs. 3,78); $F(1,103)=7,27 ; p<.008$.

Las correlaciones entre las variables edad y prejuicio ( $r=0,143 ; p=0,147)$ no han sido significativas entre los participantes. Tampoco hay diferencias significativas $(t=$ 0,794; $g l=103 ; p=0,429$ n.s.) entre los sujetos, respecto a las variables ocupación y prejuicio.

Según puede observarse en la Tabla 2, la intensidad de las emociones suscitadas hacia los afrobrasileños, en general, es baja, siendo en todo caso mayores las emociones positivas que negativas. De hecho, dos emociones superan la media teorica de la escala (3). Concretamente se trata de las emociones positivas: cordialidad y simpatía, además de admiración que se encuentra en la media de los sentimientos despertados por los negros y mestizos. Si considerarmos las emociones negativas, el sentimiento de hostilidad es el que evoca, en mayor medida, las emociones negativas hacia los afrobrasileños seguidos de desconfianza e inseguridad. Asimismo, podemos ver que la emoción negativa hostilidad que tradicionalmente está asociada con el prejuicio, es relativamente baja con relación a la media de la escala, de la misma manera que las otras emociones negativas "no tradicionales"(indiferencia, desconfianza, inseguridad y incomodidad).

Tabla 2

Media de las Emociones Evocadas por los Afrobrasileños en la Escala de Emociones utilizada por Rueda y Navas (1996)

\begin{tabular}{lll}
\hline & Media & Desviación típ. \\
\hline Admiración & 2,99 & 1,251 \\
Ódio & 1,38 &, 954 \\
Atracción & 2,40 & 1,335 \\
Hostilidad & 2,08 & 1,169 \\
Miedo & 1,50 & 1,075 \\
Envidia & 1,22 &, 835 \\
Simpatía & 3,26 & 1,381 \\
Incomodidad & 1,40 &, 838 \\
Rabia & 1,34 &, 886 \\
Desconfianza & 1.83 & 1,301 \\
Indiferencia & 1,47 & 1,135 \\
Cordialidad & 3,09 & 1,464 \\
Pena & 1,56 & 1,072 \\
Inseguridad & 1,62 & 1,178 \\
\hline$N=105$ & &
\end{tabular}

Estos resultados ponen de manifiesto que la intensidad de las emociones negativas despertadas por los afrobrasileños en la muestra estudiada es bastante baja. Estos resultados encontrados están en consonancia con los postulados de los modelos de las nuevas manifestaciones del prejuicio racial. Según estas teorías, el aspecto emocional del prejuicio es quizá, el aspecto más innovador y, precisamente, una de las características de ello consiste en evitar la manifestación de los sentimientos abiertamente hostiles hacia el exogrupo. De acuerdo con esto, Dovidio y Gaertner (1986) señalan que el sentimiento negativo hacia los negros no se expresa en hostilidad u ódio, sino en incomodidad, inseguridad y, a veces, miedo.

\section{Análisis Factorial}

A continuación, el análisis factorial (método de componentes principales con rotación Varimax) realizado sobre las respuestas a la escala de emociones (Rueda \& Navas, 1996), facilitó como resultado la identificación de 4 factores. Los dos primeros factores explican en conjunto un 47, $8 \%$ de la varianza total de la escala. Los factores y los item que saturan en cada factor aparecen recogidos en la Tabla 3.

El primer factor, que explica el 32,86 \% de la varianza total está formado por la mayoría de las emociones negativas (ódio, miedo, incomodidad, rabia, inseguridad, desconfianza y indiferencia). El factor 2 explica un 14,94\% de la varianza y agrupa las emociones positivas (admiración, atracción, simpatía y cordialidad) 
Pires, A. M. L. T. e Alonso, F. M. "El prejuicio racial en Brasil: nuevas medidas y perspectivas"

Tabla 3

Factores de la Escala de Emociones

\begin{tabular}{|c|c|c|c|c|c|}
\hline \multicolumn{2}{|l|}{ Factor 1} & \multicolumn{2}{|l|}{ Fact or 2} & Factor 3 & Factor 4 \\
\hline \multicolumn{2}{|c|}{$\begin{array}{l}\text { Varianza explicada } \\
(32,86 \%) \\
\text { Items }\end{array}$} & \multicolumn{2}{|c|}{$\begin{array}{l}\text { V arianza explicada } \\
(14,94 \%) \\
\text { Items }\end{array}$} & $\begin{array}{l}\text { Varianza explicada } \\
(8,15 \%) \\
\text { Item }\end{array}$ & $\begin{array}{l}\text { Varianza explicada } \\
(8,42 \%) \\
\text { Item }\end{array}$ \\
\hline Ódio & .63 & Admiración & .65 & Invidia .88 & Pena .77 \\
\hline Miedo & .72 & Atracción & .62 & & \\
\hline Incomodidad & .82 & Símpatía & .71 & & \\
\hline Rabia & .86 & Cordialidad & .71 & & \\
\hline Ins eguridad & .76 & & & & \\
\hline Desconfianza & .80 & & & & \\
\hline Indiferencia & .82 & & & & \\
\hline
\end{tabular}

\section{Conclusiones del Primer Estudio}

evocadas por los afrobrasileños en el grupo en cuestión. Finalmente el tercer y cuarto factores, que explican un 8,57 y 8,42 respectivamente de la varianza total, recogen las emociones de invidia y pena.

Una vez realizado este análisis factorial, y tomando unicamente los factores 1 e 2 , obtuvimos la fiabilidad de estos factores que definen las emociones positivas y negativas. A partir de los resultados de toda la muestra, la fiabilidad del factor 1 es alta (Alpha de Cronbach $=, 89$ ) y para el factor 2, el Alpha de Cronbach es ,65.

Con el fin de analizar la posible diferencia entre los sujetos con relación al prejuicio y las emociones suscitadas, creamos dos grupos de sujetos utilizando como criterio de agrupación la media de la escala de Racismo Moderno (3.49) y comparamos las puntuaciones obtenidas en los factores 1 e 2 (emociones positivas y negativas) a través de la prueba $t$ para muestras independientes. Los resultados presentamos en la Tabla 4.

Por lo que se puede observar, hay una diferencia estadísticamente significativa entre los grupos "alto" y "bajo" en prejuicio con respecto a las emociones positivas $t=(103)-2,16 ; p<0,03$ evocadas por los los afrobrasileños en los sujetos de la muestra. Como era de esperar, los participantes con puntuaciones menores en prejuicio, son los que experimentan en menor grado las emociones negativas y en mayor grado las emociones positivas, siendo menor la diferencia entre los grupos "alto y "bajo" con relación a las emociones negativas. De esta manera, los sujetos "altos" en prejuicio, manifiestan menor disposición en expresar los sentimientos negativos hacia al exogrupo.
El objetivo del primer estudio era analizar las manifestaciones de prejuicio en sujetos pertenecientes al grupo "blanco". A la luz de los resultados obtenidos podemos destacar que el prejuicio declarado hacia los afrobrasileños es relativamente bajo en la muestra estudiada. Las mujeres declararon sentir menos prejuicio hacia los afrodescendientes que los hombres.

Igualmente es importante destacar la baja intensidad de las emociones suscitadas hacia los afrobrasileños. Merece la pena señalar que la intensidad de las emociones positivas (cordialidad, simpatía, admiración) son mayores que las que negativas (hostilidad desconfianza y inseguridad) en los sujetos participantes en nuestro estudio.

Encontramos también que, con relación a la manifestación de las emociones positivas hay diferencias significativas entre los sujetos con mayor y menor puntuación en prejuicio. Son los sujetos con menor puntuación en prejuicio los que experimentan en mayor grado las emociones positivas, pero cuando se trata de la expresión de las emociones negativas, la diferencia entre los dos grupos "alto" y "bajo" en prejuicio es menor. Los sujetos se muestran menos dispuestos a expresar sentimientos negativos hacia los negros y mestizos, encontrándose una pauta moderada de la expresión de los sentimientos que generan en ellos los afrobrasileños.

Por tanto, los resultados de nuestro estudio, están en general, de acuerdo con los encontrados en la literatura existente sobre el prejuicio y apoyan los modelos de racimo moderno. Desde esta perspectiva, la nueva expresión del sentimiento negativo hacia miembros de otras razas se traduce en una forma más sutil y racionalizada de expresar el sentimiento racial negativo. 
Tabla 4

Diferencias entre los Sujetos Bajos y Altos en Prejuicio con Respecto a las Emociones que les producen los Afrobrasileños

\begin{tabular}{lccccc}
\hline & $\begin{array}{l}\text { Altos } \\
\text { prejuicio }\end{array}$ & \multicolumn{3}{c}{$\begin{array}{l}\text { Bajos } \\
\text { prejuicio }\end{array}$} \\
& Media & Des. Tip & Media & Desv. Típic & Sig.(bilateral) \\
&,- 19 &, 86 &, 21 & 1,10 & $P<0,03$ \\
$\begin{array}{l}\text { Emociones } \\
\text { positivas }\end{array}$ &, 18 &, 96 &,- 19 & 1,00 & $P<0,05$ \\
$\begin{array}{l}\text { Emociones } \\
\text { negativas }\end{array}$ & & & & & \\
\hline
\end{tabular}

\section{Estudio 2}

\section{Percepción del Prejuicio en Sujetos de la Raza Negra/Mestiza}

El estudio tuve el objetivo de medir el grado de prejuicio general y discriminación personal percibidos por sujetos negros y mestizos y analizar las diferencias en los dos grupos.

\section{Muestra}

En el segundo estudio han participado un total de 101( 60 mujeres y 41 hombres). El 45,5\% de la muestra declaró pertenecer al grupo de personas de la raza negra y el 54,5\% se autoclasificaron como mestizos. La media de edad era de 20,74, con un rango de edades que osciló entre 16 y 50 años. La mayoría de los sujetos $(68,3 \%)$ trabaja y estudia, estando en menor porcentaje el grupo que solo estudia $(31,7 \%)$.

\section{Procedimiento y Instrumentos}

Los sujetos que participaran en este estudio, contestaron un cuestionario que incluía las seguintes siguientes secciones:

1. Características sociodemográficas: edad, sexo y ocupación, además de indicar la raza de pertenencia.

2. Una medida diseñada para medir la percepción del prejuicio en los sujetos. Los ítems se diseñaron a partir de la literatura revisada sobre prejuicio, adaptándose a los objetivos de la investigación. Esta escala constaba de 10 ítems. De ellos, 6 ítems para medir la percepción general de rechazo con un formato de respuesta tipo Likert de 7 puntos y otros 4 ítems para medir la percepción personal de discriminación con un rango de respuestas comprendidas entre $1=$ nunca; $5=$ muy frecuente. Estos ítems se han extraído de la literatura y adaptados a los objetivos del estudio.

\section{Resultados}

\section{Fiabilidad y Estadísticos Descriptivos}

En primer lugar, con el fin de analizar la fiabilidad de las escalas, realizamos análisis de fiabilidad Alpha de Cronbach de cada una de ellas. La escala de percepción general de prejuicio tiene un coeficiente Alpha de .58 y la escala de discriminación el coeficiente .72. Se hallaron también la media total de las escalas. Se presentan los resultados en la Tabla 5.

Tabla 5

Estadísticos Descriptivos y Fiabilidad de las Escalas

\begin{tabular}{|c|c|c|}
\hline & $N=101 \quad$ Alpha & Media total \\
\hline Percepción general de prejuicio & 58 & 4,73 \\
\hline Discriminación personal percibida & .72 & 1,94 \\
\hline
\end{tabular}

Como puede verse en la Tabla 5, los sujetos de la muestra tienen una percepción general de prejuicio algo superior a la media teórica de la escala (4) en un rango de respuestas de 1 a 7 , y por el contrario, declaraban no haber padecido personalmente mucha discriminación 1,94 (puntuación ligeramente inferior al 2 sobre un máximo de 5). Esta diferencia es significativa: $t(100)=-12.11$; $p<0001$ con respecto al punto medio de la escala (3).

Asimismo y con el objetivo de averiguar las posibles diferencias entre las variables en estudio y el sexo de los sujetos de la muestra, hicimos una prueba $t$ para muestras independientes. Los resultados no han sido significativos: $t(99)=-, 233 ; p>0,05$ y $t(99)=-1,81 ; p>0,05$ entre los participantes. Tampoco hay diferencias significativas entre las variables ocupación y la percepción de prejuicio y discriminación.

Conel fin de analizar la posible diferenciaentre la variable etnia, la de percepción de general de prejuicio y discriminación personal, hicimos una prueba $t$ de muestras independientes. Los resultados se puede observar en la Tabla 6 . 
Pires, A. M. L. T. e Alonso, F. M. "El prejuicio racial en Brasil: nuevas medidas y perspectivas"

Tabla 6

Diferencias entre Etnia, Percepción de General de Prejuicio y Discriminación Personal Percibida

\begin{tabular}{llllll}
\hline Escala & Etnia & $\mathrm{N}$ & Media & Desv. Típ. & Sig. \\
\hline Percep.General de prejuicio & Negros & 46 & 4,83 & 1,14 & n.s. \\
& Mestizos & 55 & 4,64 & 1,10 & \\
Percep. discr. Personal & Negros & 46 & 2,21 &, 87 & $t(99)=2,96 p<.004$ \\
& Mestizos & 55 & 1,71 &, 81 & \\
\hline
\end{tabular}

Como podemos observar, la Tabla 6 muestra una diferencia significativa entre los grupos negro y mestizo con relación a la discriminación personal percibida $t(99)$ $=2,96 ; p<0,04$. Con relación a la percepción de discriminación personal, existe una diferencia entre los sujetos negros y mestizos de la muestra. Los sujetos que se dicen de la raza negra se sienten personalmente más discriminados en comparación con los sujetos que se declaran mestizos. Respecto a la percepción general de rechazo no produjo resultados significativos entre los grupos $t(99)=, 854 ; p>0,05$.

\section{Conclusiones del Segundo Estudio}

El objetivo de nuestro segundo estudio era conocer la percepción de prejuicio de las personas pertenecientes a la de raza negra/mestiza hacia su grupo y la discriminación que personalmente ellas han padecido. A la vista de los resultados obtenidos podemos destacar que los participantes en nuestro estudio perciben un prejuicio general medio hacia su grupo y que la frecuencia en la que han sido discriminados personalmente ha sido baja. El prejuicio que los sujetos perciben en general hacia su grupo es menor que la percepción de discriminación que ellos han sufrido personalmente. Resultados similares se encuentran en la literatura existente sobre el prejuicio. Encontramos asimismo una diferencia estadísticamente significativa entre la percepción de discriminación personal que han padecido y la variable etnia, siendo mayor la discriminación percibida en los sujetos que se autoidentificaron como negros en comparación a los sujetos que se han autoidentificado como mestizos.

\section{Discusión y Conclusiones}

Uno de los objetivos de nuestro estudio era adaptar la escala de racismo moderno de McConahay et al. (1981) a la población brasileña. Los primeros resultados obtenidos nos permiten decir que la escala parece ser un instrumento útil para medir el prejuicio hacia los afrobrasileños. Así lo demuestra el coeficiente de fiabilidad obtenido que a pesar de no ser muy alto, podemos considerar aceptable. Otro de nuestros objetivos principales era analizar la manifestación del prejuicio en las personas de raza blanca.
A la vista de los resultados, es importante destacar los niveles bajos de prejuicio hacia los afrobrasileños encontrados en los participantes de nuestro estudio. Es evidente que hay que tener en cuenta por un lado, la muestra con la que hemos trabajado, no sólo por tu tamaño, sino también por su homogeneidad en lo que se refiere a algunas variables como la edad, el nivel de estudios y socioeconómico, que aparecen tradicionalmente relacionadas en la literatura sobre el tema con niveles bajos de prejuicio. Por otra parte, estos resultados pueden estar también asociados a la fuerte indeseabilidad social al hecho de mostrarse racista, como lo han demostrado varios estudios. Debemos aquí considerar que, a pesar de que los ítems de la escala de McConahay sean menos reactivos, no están libres del sesgo de deseabilidad social común en estos tipos de escalas diseñadas para medir actitudes en los sujetos. Con relación a otras investigaciones en que se ha utilizado la misma escala, los hallazgos resultan de gran interés en relación a esta metodología, pero es importante tener en cuenta las peculiaridades del grupo y del contexto en cuestión (véase Lepore \& Brow,1994, cit. en Brown, 1995; Augoustinos, Ahrens, \& Innes, 1994; Navas, 1998). A nuestro juicio, el bajo nivel de prejuicio hacia los negros y mestizos, alude también a la influencia de las variables contextuales relacionadas a la cuestión histórica del prejuicio en el entorno social brasileño (véase Turra \& Venturi, 1995; Martinez \& Camino, 2000).

Dentro de los objetivos del presente estudio nos planteábamos conocer la percepción de prejuicio y la discriminación personal que sienten las personas de raza negra/ mestiza. Los resultados de nuestra muestra indican un nivel medio de percepción general de prejuicio hacia su grupo y la frecuencia con que han sido discriminados personalmente ha sido baja. El prejuicio que los sujetos perciben, en general, hacia su grupo es menor que la percepción de discriminación que ellos han sufrido personalmente. Estes resultados coinciden con los que se obtiene en la literatura sobre el prejuicio. Taylor, Wright, Moghaddan y Lalonde (1990) argumentan que los componentes de grupos desfavorecidos distinguen entre dos niveles de discriminación: uno personal y uno grupal, de tal forma que, normalmente, perciben más discriminación 
contra su grupo que contra su persona. Otro acercamiento sobre el tema sugiere que los componentes de grupos desfavorecidos rechazan o "minimizan" la discriminación personal, como estrategia defensiva ante ella (Allport, 1954). En esta misma línea, los resultados de los estudios de Ruggiero (1999), con grupos minoritarios (mujeres y afroamericanos) muestran un reconocimiento mayor de la discriminación grupal que la discriminación personal. Para estos autores "la tendencia de los componentes de las minorías a minimizar la discriminación personal puede llevarles a restar importancia a la discriminación real contra su grupo. Sin embargo, como estrategia defensiva tiene consecuencias psicológicas positivas para la víctima del prejuicio, ya que creer que tienen control sobre sus resultados repercute de forma positivamente en su autoestima" (véase Ruggiero \& Taylor, 1997).

Otros resultados de nuestra investigación, derivados de la comparación entre etnia y la percepción de discriminación personal entre los participantes, ponen de manifiesto que hay una mayor discriminación percibida en los sujetos que se autoidentificaron como negros en comparación a los sujetos que se autoidentificaron como mestizos. Para Camino, Silva, Machado y Pereira (2001) el racismo en el contexto brasileño está relacionado con la pobreza y, el color de la piel, es el punto central en la definición de la clase social de pertenencia de los individuos. En este sentido, queda demostrado en nuestro estudio que las experiencias negativas de discriminación, relacionadas con el color de la piel, están influyendo en la percepción de rechazo.

Finalmente, cabe preguntarse por qué los afrobrasileños, en general, no se sienten discriminados a pesar de existir indicadores objetivos de discriminación. Esta cuestión nos lleva a reflexionar sobre las situaciones de interacción ambiguas de discriminación. Esta paradoja expresa bien, a nuestro juizio, el carácter de la naturaleza ambigua y normalmente sutil de la mayoría de las conductas discriminativas, y pone de manifiesto, de acuerdo con los estudios de Ruggiero (1999), que la sobreestimación y la subestimación de las conductas de discriminación son frecuentes entre los grupos discriminados y ambas cumplen una función adaptativa de autoprotección frente a las conductas discriminativas.

\section{Referencias}

Allport, G. W. (1954). The nature of prejudice. Reading, MA: Addison Wesley.

Augoustinos, M., Ahrens, C., \& Innes, J. M. (1994). Stereotypes and prejudice: The Australian experience. British Journal of Social Psychology, 33, 125-141.

Azevedo, T. (1975). Democracia racial. Rio de Janeiro, RJ: Vozes. Bastide, R. (1956). Stereotypes et prejuge de couleur. Sociologia: Revista Didática e Científica da Escola Livre de Sociologia e Política, 18(2), 141-171.
Bastide, R., \& Fernandes, F. (1959). Brancos e negros em São Paulo: Ensaio sociológico sobre aspectos da formação, manifestações atuais e efeitos do preconceito de cor na sociedade paulistana: Vol. 305. Brasiliana. São Paulo, SP: Nacional.

Bastide, R., \& van den Berghe, P. (1957). Stereotypes, norms, and interracial behavior in São Paulo, Brasil. American Sociological Review, 22, 689-694.

Brown, R. J. (1995). Prejudice: Its Social Psychology. Oxford, UK: Blackwell.

Camino, L., Silva, P., Machado, A., \& Pereira, C. (2001). A face oculta do racismo no racismo no Brasil: Uma análise psicosociológica. Revista de Psicologia Política, 1, 13-36.

Dovidio, J. F., \& Gaertner, S. L. (1986). The aversive form of racism. In J. F. Dovidio \& S. L. Gaertner (Eds.), Prejudice, discrimination and racis (pp. 61-89). New York: Academic Press.

Duckitt, J. (1992). A historical analysis and integrative framework. American Psychologist, 47, 1182-1193

Echebarría, A., \& Villarreal, M. (1995). Psicología Social del racismo. In A. Echebarría, M. T. Garaigordobil, J. L. González, \& M. Villarreal (Eds.), Psicología Social del prejuicio y el racismo (Cap. 10). Madrid, España: Ramón.

Fernandes, F. (1972). O negro no mundo dos brancos. São Paulo, SP: Difel.

Fernandes, F., \& Bastilde, R. (1959). Brancos e negros em São Paulo. São Paulo, SP: Companhia Editora Nacional.

Freyre, G. (1933). Casa Grande e Senzala: Formação da família brasileira sob o regime da economia patriarcal. Rio de Janeiro, RJ: Maia \& Schmidt.

Fry, P. (2005). A persistência da raça: Ensaios antropológicos sobre o Brasil e a África austral. Rio de Janeiro, RJ: Civilização Brasileira.

Guimarães, A. S. A. (1999). Racismo e anti-racismo no Brasil. São Paulo, SP: Ed. 34.

Hasenbalg, C. A., \& Silva, N. (1992). Relações raciais no Brasil contemporâneo. Rio de Janeiro, RJ: Rio Fundo.

Instituto Brasileiro de Geografia e Estatística. (2000). Encuesta nacional de muestras por hogares. Brasília, DF: Autor. Retrieved from http://www.ibge.gov.br

Instituto Brasileiro de Geografia e Estatística. (2005). Sintese de Indicadores Sociais 2005. Brasília, DF: Autor. Retrieved from http://www.ibge.gov.br/

Katz, I., \& Hass, R. G. (1988). Racial ambivalence and American value conflict: Correlacional and priming studies of dual cognitive structures. Journal of Personality and Social Psychology, 55, 893-905.

Kinder, D. R., \& Sears, D. O. (1981). Prejudice and politics: Symbolic racism versus racial threats to good life. Journal of Personality and Social Psychology, 40, 414-431.

Maggie, Y., \& Gonçalves, M. A. (1995). Pessoas fora do lugar: A produção da diferença no Brasil. In M. A. Gonçalves \& G. Villas Boas (Eds.), O Brasil na virada do século. Rio de Janeiro, RJ: Relume-Dumará.

Martinez, I., \& Camino, L. (2000). Brasil es racista, Brasil no es racista: el discurso social como determinante de las diferencias percibidas entre blancos, negros y morenos. Comunicação apresentada no VII Congresso de Psicologia Social da Espanha, Oviedo.

McConahay, J. B. (1986). Modern racism, ambivalence and the modern racism scale. In J. F. Dovidio \& S. L. Gaertner (Eds.), Prejudice, discrimination and racism (pp. 91-126). New York: Academic Press. 
McConahay, J. B., Hardee, B. B., \& Batts, V. (1981). Has racism declined in America? It depends upon who is asking and what is asked. Journal of Conflict Resolution, 25, 563-579.

McConahay, J. B., \& Hought, J. C., Jr. (1976). Symbolic racism. Journal of Social Issues, 32, 23-45.

Navas, M. S. (1998). Nuevos instrumentos de medidapara el nuevo racismo. Revista de Psicología Social, 13(2), 233-239.

Nascimento, A. (1968). O negro revoltado. Rio de Janeiro, RJ: Nova Fronteira.

Pedersen, A., \& Walker, I. A. (1997). Prejudice against Australian aborigines: Old-fashioned and modern forms. European Journal of Social Psychology, 27, 561-587.

Pettigrew, T. F., \& Meertens, R.W. (1995). Subtle and blatant prejudice in Western Europe. European Journal of Social Psychology, 25, 57-75.

Rueda, J. F., \& Navas, M. (1996). Hacia una evaluación de las nuevas formas del prejuicio racial: las actitudes sutiles del racismo. Revista de Psicología Social, 11(2), 131-149.

Ruggiero, K. M. (1999). The personal/group discrimination discrepancy: Extending Allport's analysis of targets. Journal of Social Issues, 55(3), 519-536.

Ruggiero, K. M., \& Taylor, D. M. (1997). Why minority group members perceive or do not perceive the discrimination that confronts them: The role of self-esteem and perceived control. Journal of Personality and Social Psychology, 72, 373-389.

Sears, D. O. (1992). Conflicto político y política de la raza en los Estados Unidos. Psicología Política, 5, 71-98.

Taylor, D. M., Wright, S. C., Moghaddam, F. M., \& Lalonde, R. N. (1990). The personal/group discrimination discrepancy: Perceiving my group, but not my self, to be a target for discrimination. Personality and Social Psychology Bulletin, 16, 254-262.

Turra, C., \& Venturi, G. (1995). Racismo cordial: A mais completa análise sobre preconceito de cor no Brasil. São Paulo, SP: Ática.

Walker, I. (1994). Attitudes toward minorities: Survey evidence of the Western Australians attitudes to Aborigenes, Asians und women. Australian Journal of Psychology, 46, 41-56.

Ana Maria Lacerda Teixeira Pires - Facultad de Psicología de la Universidad Nacional de Educación a

Distancia (UNED), Madrid, España. anamariapires@hotmail.com

Fernando Molero Alonso es profesor titular de Psicología Social en la UNED.

Dirección: Universidad Nacional de Educación a Distancia, Facultad de Psicologia, Departamento de Psicologia Social y de las Organizaciones, C/ Juan del Rosal, Madrid, España, 1028040. fmolero@psi.uned.es

El Prejuicio Racial en Brasil:

Nuevas Medidas y Perspectivas

Ana Maria Lacerda Teixeira Pires e Fernando Molero Alonso

Recebido: 26/02/2008

Aceite final: 12/07/2008 